\title{
Diferenças entre o Método Trecho-a-Trecho e o Método de Múltiplas Saídas na determinação das perdas de cargas em uma linha lateral de um sistema de irrigação localizada
}

\section{Eugenio Paceli de Miranda ${ }^{1}$, Gilbenes Bezerra Rosal ${ }^{2}$, Francisco Uchoa de Lima ${ }^{2}$}

${ }^{1}$ Instituto Federal de Educação, Ciência e Tecnologia do Ceará - IFCE. Campus Iguatu. Rodovia Iguatu-Várzea Alegre, km 05, S/Nº Vila Cajazeiras. Iguatu-CE, Brasil (CEP 63500-000).E-mail: eu.paceli@yahoo.com.br.

${ }^{2}$ Curso de Graduação em Tecnologia em Irrigação e Drenagem. Instituto Federal de Educação, Ciência e Tecnologia do Ceará - IFCE. Campus Iguatu. Rodovia IguatuVárzea Alegre, km 05, S/Nº. Vila Cajazeiras. Iguatu-CE, Brasil (CEP 63500-000).

Resumo. A perda de carga é o parâmetro fundamental para o dimensionamento dos sistemas de irrigação. Sua determinação correta tem relação com os custos dos materiais e com o consumo de energia. Este trabalho teve como objetivo comparar a determinação das perdas de cargas em uma linha lateral de um sistema de irrigação localizada por microaspersão, usando o método de múltiplas saídas e o método trecho-a-trecho. No dimensionamento alterou-se o comprimento da linha lateral, aumentando consequentemente o número de aspersores e a vazão da linha lateral e a pressão de serviço, aumentando com isso a vazão da linha lateral e mantendo o mesmo comprimento e número de microaspersores por lateral. Tanto para o aumento do comprimento da linha lateral como para o aumento da pressão de serviço, a perda de carga determinada pelo método trecho-atrecho foi maior que o método de múltiplas saídas, a maior diferença observada foi de 8,8\%. A diferença entre os dois métodos aumentou a medida que o comprimento da linha lateral aumentou. 0 contrário aconteceu com o aumento da pressão de serviço, que provocou uma redução nas diferenças entre os dois métodos.

Palavras-chave: Perda de carga; Equação de Darcy-Weisbach; Coeficiente de Uniformidade de Christiansen.

Abstract. Comparison between the Step-by-Step Method and the Multiple Output Method in the determination of head losses line of an microirrigation system. The head loss is the fundamental parameter for the design of irrigation systems. Its correct determination relates to material costs and energy consumption. The objective of this study was to compare the determination of head losses of a lateral line of an irrigation system located by microsprinkler using the multiple output
Recebido:

$18 / 09 / 2018$

Aceito:

$29 / 11 / 2018$

Publicado:

$31 / 12 / 2018$

Acesso aberto

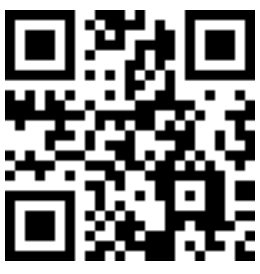

ORCID

D 0000-0002-1390-1639

Eugenio Paceli de Miranda

D 0000-0002-1871-7195 Gilbenes Bezerra Rosal

D 0000-0002-1040-0804

Francisco Uchoa de

Lima 
method and the step-by-step method. In the design, the length of the lateral line was changed, consequently increasing the number of sprinklers and the lateral line flow and the operating pressure, thus increasing the lateral line flow and maintaining the same length and number of microsprinklers per line. For both the increase of the lateral line length and the increase of the service pressure, the head loss determined by the Step-by-Step Method was greater than the Multiple Output Method, the largest difference observed was $8.8 \%$. The difference between the two methods increased as the lateral line length increased. The opposite happened with the increase in the service pressure, which caused a reduction in the differences between the two methods.

Keywords: Head loss; Darcy-Weisbach's Equation; Christiansen's Uniformity Coefficient.

\section{Introdução}

De acordo com Alves et al. (2015) a irrigação se caracteriza como uma tecnologia que está frequentemente apresentando inovações, capazes de proporcionar elevados níveis de desempenho dos sistemas, nas mais adversas e diversas condições; isso é proveniente de pesquisas, que promovem o desenvolvimento da ciência de irrigação, numa procura constante do melhor resultado aliado ao menor custo, por isso a busca por estratégias para otimizar a eficiência de aplicação de água tem sido objeto de estudo de vários pesquisadores.

Sabe-se que para o dimensionamento do sistema de irrigação é necessário o conhecimento de dados técnicos específicos do comportamento hidráulico do líquido e também referente ao sistema água-solo-planta-atmosfera da região. 0 tipo de líquido e consequentemente a sua perda de carga em tubulações são fundamentais no dimensionamento das tubulações e das bombas desses sistemas, uma vez que a altura manométrica representa a energia fornecida ao líquido suficiente para vencer o desnível geométrico e as perdas de carga ocorridas ao longo do percurso (Sampaio et al., 2007).

A utilização de sistemas de microaspersão, devido ao seu baixo custo e elevada uniformidade de aplicação de água, vem sendo uma boa alternativa para pequenos agricultores, porém, para que sejam atingidas boas uniformidades de aplicação de água é imprescindível que o sistema seja bem dimensionado, ou seja, é necessário um projeto criterioso e detalhado (Alves et al., 2015).

Segundo Bombardelli (2018) o grande interesse pela microaspersão foi despertado principalmente pelos resultados de economia de água e energia que o sistema pode oferecer, aliado ao grande potencial para oferecer condições ótimas de manejo para o desenvolvimento e produtividade das plantas; uma vez que o objetivo final de qualquer atividade agrícola é atingir o máximo retorno econômico líquido.

Melo (2018) ressalta que, muitas das vezes estes sistemas deixam de ser eficientes quanto ao uso da água, gerando desperdício devido a aplicação excessiva da mesma ou por nulidade do sistema de irrigação adotado, ocasionado pelo vazamento de tubulações ou pela baixa uniformidade de distribuição, neste caso existe a necessidade de estudos que otimizem esta técnica, bem como seu manejo.

As linhas laterais de um sistema de microaspersão são condutos pressurizados que distribuem a água através dos emissores, os quais mantém 
uma uniformidade de distribuição em função da variação de pressão na linha. A introdução de componentes adicionais, tais como conexões, válvulas, cotovelos, curvas, tês, emissores inseridos na linha lateral e outras possíveis espécies de excentricidades, interferem numa perda de carga pontualizada na tubulação, com alterações bruscas de escoamento do fluido, em módulo e direção (Bombardelli, 2018).

Sampaio et al. (2007) relata que essa perda de carga localizada tem como causa a turbulência do fluido, gerada por essas peças especiais colocadas ao longo da tubulação, sempre necessárias para realizar qualquer controle, e que a precisão na determinação dessa perda de carga influenciará nos custos finais do projeto, pois o custo da tubulação está associado diretamente com o seu diâmetro e material. 0 mesmo autor afirma ainda que os agentes causadores dessa perda de carga, são os atritos "interno", que é uma consequência da viscosidade do fluido, e aqueles decorrentes do contato entre o fluido e as paredes dos condutos.

Como a determinação da perda de carga em campo torna-se inviável ao projetista para o dimensionamento hidráulico, logo existem diversas equações que estimam a perda de carga nas tubulações (Coelho et al., 2018).

Como a perda de carga é dependente de diversos fatores, a utilização de equações para sua estimativa é uma tarefa difícil e que muitas vezes não representa a realidade (Alazba et al., 2012).

Geralmente as equações superestimam as perdas de carga que ocorrem, pois as tubulações de polietileno, mais comumente utilizadas em sistemas de irrigação, aumentam seu diâmetro quando pressurizadas, diminuindo a perda de carga que é determinada (Rettore Neto et al., 2013).
Dentre os métodos para estimar a perda de carga, tem-se a Equação de Darcy-Weisbach, de 1985, como sendo a mais aceita pela comunidade científica, ela também é conhecida como Fórmula Universal, foi deduzida através de investigações acerca da tensão da água sob as paredes da canalização e lei de semelhança, cujo princípio é baseado pela análise dimensional, por conta disso é considerada de base teórica (Bombardelli, 2018).

De acordo com Rettore Neto et al. (2013) a Equação de Darcy-Weisbach, com uso do cálculo do fator de atrito por Blasius, obtem-se menor variação dos valores comparando com os determinados em laboratório.

Ainda segundo Rettore Neto et al. (2014) é possível minimizar a diferença dos métodos de estimativa da perda de carga, porém necessita de conhecimento especifico dos profissionais pela complexidade e informações muito específicas dos materiais das tubulações como coeficientes de elasticidade, valores que nem sempre são facilmente disponíveis.

No presente trabalho o objetivo foi comparar o método trecho-a-trecho com o método de múltiplas saídas na determinação das perdas de cargas.

\section{Material e métodos}

Determinou-se a perda de carga em uma linha lateral de um sistema de irrigação localizada do tipo microaspersão utilizando o método trecho-a-trecho e o método de múltiplas saídas, variando o comprimento da linha lateral e a pressão de serviço do emissor. O microaspersor utilizado foi o Modelo OPAL (NaanDaanJain), funcionando com uma pressão de serviço de 20 mca e espaçados de $5 \mathrm{~m}$. As características hidráulicas do microaspersor e da linha lateral são mostradas na Tabela 1. 
Tabela1. Características hidráulicas do microaspersor e da linha lateral Equação da vazão do aspersor (q - vazão (L.h-1 ); H - pressão (mca); k - coeficiente matemático; x - expoente de descarga).

\begin{tabular}{lc}
\hline Parâmetro & Característica \\
\hline Emissor & Microaspersor \\
Modelo & OPAL \\
Fabricante & NaanDaanJain \\
Equação da vazão & $\mathrm{Q}=6,7 \mathrm{H}^{0,5}$ \\
Espaçamento $(\mathrm{m})$ & 5,0 \\
Diâmetro da linha lateral $(\mathrm{m})$ & 15,3 \\
Temperatura da água $\left({ }^{\circ} \mathrm{C}\right)$ & 25 \\
Pressão de serviço (mca) & 20 \\
\hline
\end{tabular}

Usou-se a Equação de DarcyWeisbach (Equação 1) para determinar a perda de carga contínua para o método trecho-a-trecho. Para o método de múltiplas saídas, a Equação de DarcyWeisbach foi corrigida (Equação 2) utilizado o Fator de Ajuste de Christiansen (Equação 3).

$$
\begin{aligned}
& h f=f \frac{L}{D} \frac{v^{2}}{2 g} \\
& h f=f \frac{L}{D} \frac{v^{2}}{2 g} \cdot F n
\end{aligned}
$$

$$
F n=\frac{1}{m+1}+\frac{1}{2 N}+\frac{\sqrt{m-1}}{6 N^{2}}
$$

Em que: hf - Perda de carga (m); f - Fator de atrito de Darcy-Weisbach (adimensional); L - Comprimento da tubulação (m); D - Diâmetro interno da tubulação (m); $\mathrm{v}$ - Velocidade de escoamento $\left(\mathrm{m} \mathrm{s}^{-1}\right)$; g - Aceleração da gravidade ( $\left.\mathrm{m} \mathrm{s}^{-2}\right)$; Fn - Fator de correção de múltiplas saídas; $\mathrm{m}$ - expoente da velocidade; $\mathrm{N}$ - número de aspersores.

Usou-se a equação da energia cinética para calcular a perda de carga localizada (Equação 4) para o método trecho-a-trecho e a Equação 5 para o método de múltiplas saídas.

$$
\begin{aligned}
& \mathrm{hf}_{\text {Loc }}=\mathrm{K} \cdot \frac{\mathrm{v}^{2}}{2 \mathrm{~g}} \\
& \mathrm{hf}_{\text {Loc }}=\sum \mathrm{K} \cdot \frac{\mathrm{v}^{2}}{2 \mathrm{~g}} \cdot \mathrm{Fn}
\end{aligned}
$$

Em que: $\mathrm{hf}_{\mathrm{Loc}}$ - Perda localizada de carga (m); K - Coeficiente de perda localizada de carga (adimensional); v Velocidade do fluido $\left(\mathrm{m} \mathrm{s}^{-1}\right) ; \mathrm{g} \quad$ Aceleração da gravidade $\left(\mathrm{m} \mathrm{s}^{-2}\right)$.

0 fator de atrito da Equação de Darcy-Weisbach (f) foi calculada em função do Número de Reynolds, utilizando a Equação de Hagen-Poiseuille (Equação 6) para Número de Reynolds menor que $2000(\operatorname{Re}<2000)$ e a Equação de Blasius (Equação 7), para Números de Reynolds igual ou maior que 2000 ( $R e \geq$ 2000).

$$
\mathrm{f}=\frac{64}{\operatorname{Re}}
$$

$$
f=\frac{0,316}{\operatorname{Re}^{0,25}}
$$


Em que: $f$ - Fator de atrito (adimensional); Re - Número de Reynolds (adimensional).

A determinação da perda de carga no método trecho-a-trecho (Figura 1) foi feita a partir do último emissor situado no final da linha lateral, com vazão do último emissor (qn) submetido a uma pressão $\mathrm{Hn}$, considerada como a pressão de serviço (PS), conforme Equação 8.

$$
q_{n}=k \cdot H_{n}^{x}
$$

Em que: $\mathrm{q}_{\mathrm{n}}$ - Vazão do último emissor $\left(\mathrm{Lh}^{-1}\right) ; \mathrm{k}$ - Coeficiente de proporcionalidade (adimensional); $\mathrm{H}_{\mathrm{n}}$ Pressão no último emissor (mca); x Expoente de descarga (adimensional).
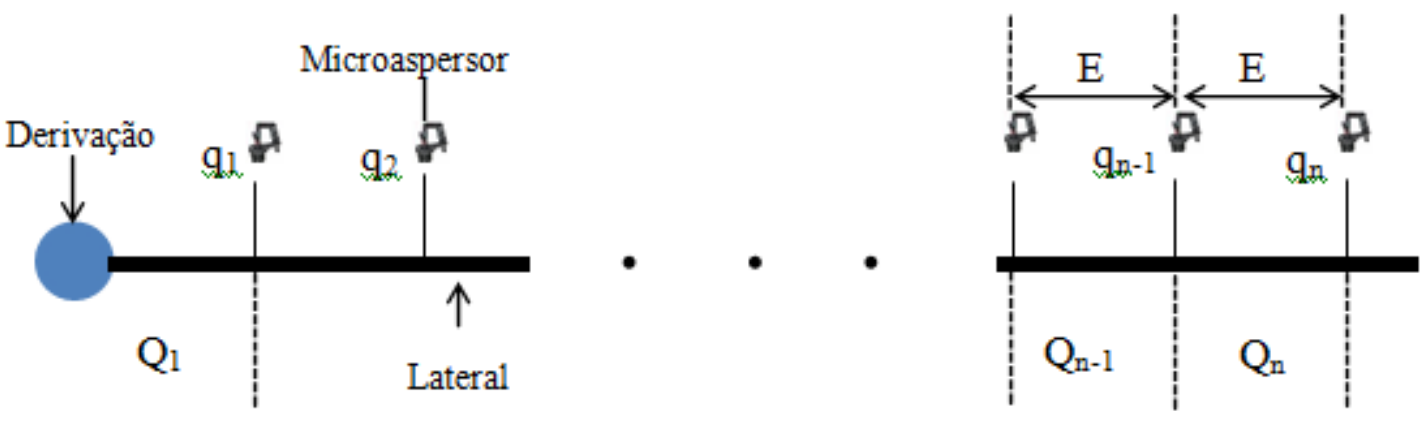

Figura 1. Esquema da disposição dos emissores e dos trechos na linha lateral $\left(\mathrm{q}_{\mathrm{n}}\right.$ - vazão do último emissor; $\mathrm{q}_{\mathrm{n}-1}$ - vazão do penúltimo emissor; $\mathrm{q}_{\mathrm{n}-2}$ - vazão do antepenúltimo emissor; $\mathrm{q}_{1}$ - vazão do primeiro emissor; $\mathrm{q}_{2}$ - vazão do segundo emissor; $Q_{n}$ - vazão do último trecho; $Q_{n-1}$ - vazão do penúltimo trecho; $Q_{1}$ - vazão do primeiro trecho; E - espaçamento entre aspersores).

A vazão do penúltimo emissor $\left(\mathrm{q}_{\mathrm{n}-1}\right)$, submetida à uma pressão $\left(\mathrm{H}_{\mathrm{n}-1}\right)$ é determinada pela Equação 9.

$$
\mathrm{q}_{\mathrm{n}-1}=\mathrm{k} \cdot \mathrm{H}_{\mathrm{n}-1}^{\mathrm{x}}
$$

Em que: $\mathrm{q}_{\mathrm{n}-1}$ - Vazão do penúltimo emissor (L.h-1); $\mathrm{k}$ - Coeficiente de proporcionalidade (adimensional); $\mathrm{H}_{\mathrm{n}-1}-$ Pressão no penúltimo emissor (m); $\mathrm{x}$ Expoente de descarga (adimensional).
A pressão do penúltimo emissor $\left(\mathrm{H}_{\mathrm{n}-1}\right)$ é determinada pela soma da pressão do último emissor $\left(\mathrm{H}_{\mathrm{n}}\right)$ com a perda de carga no último trecho $\left(\mathrm{Hf}_{\mathrm{n}}\right)$, Equação 10.

$$
\mathrm{H}_{\mathrm{n}-1}=\mathrm{H}_{\mathrm{n}}+\mathrm{Hf}_{\mathrm{n}}
$$

Em que: $H_{n-1}$ - Pressão do penúltimo emissor (mca); $H_{n}$ - Pressão do último emissor (mca); $\mathrm{Hf}_{\mathrm{n}}$ - Perda de carga do último trecho $(\mathrm{m})$; 


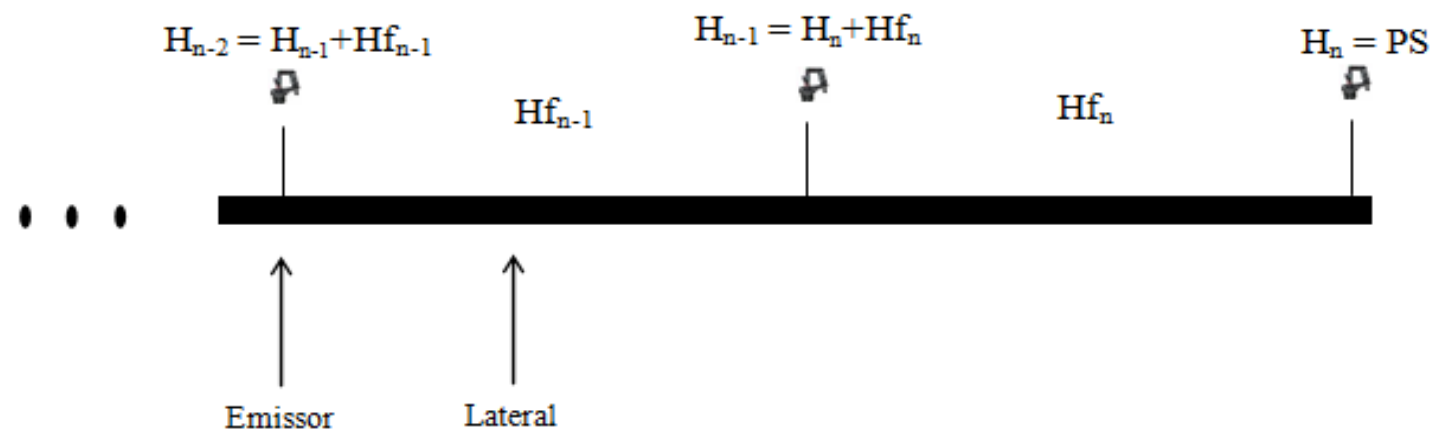

Figura 2. Esquema da determinação das pressões em cada emissor $\left(H_{n}\right.$ - pressão do último aspersor; $\mathrm{H}_{\mathrm{n}-1}$ - pressão do penúltimo aspersor; $\mathrm{H}_{\mathrm{n}-2}$ - pressão do antepenúltimo aspersor; $\mathrm{Hf}_{\mathrm{n}}$ perda de carga no último trecho; $\mathrm{Hf}_{\mathrm{n}-1}$ - perda de carga no penúltimo trecho; PS - pressão de serviço).

\section{Resultados e discussão}

As perdas de cargas para 0 Método Trecho-a-Trecho, para o Método de Múltiplas Saídas e a diferença percentual entre eles são mostradas na Tabela 2. Pode ser observado que as perdas de cargas e as diferenças entre os dois métodos aumentam a medida que aumenta o comprimento da linha lateral, chegando a $6,84 \%$ para um comprimento total de $100 \mathrm{~m}$ e 20 microaspersores. Em todas as simulações as perdas de cargas determinadas pelo método trecho-atrecho superestimou o método de múltiplas saídas.

Tabela 2. Perdas de carga em função da variação do comprimento e do número de emissores.

\begin{tabular}{lcccc}
\hline $\mathbf{L}(\mathbf{m})$ & No de emissores & Hf t-t (m) & Hf ms (m) & $\Delta$ Hf (\%) \\
\hline 50 & 10 & 1,994 & 1,994 & 0,00 \\
60 & 12 & 3,245 & 3,202 & 1,34 \\
70 & 14 & 4,923 & 4,795 & 2,67 \\
80 & 16 & 7,097 & 6,818 & 4,09 \\
90 & 18 & 9,840 & 9,316 & 5,62 \\
100 & 20 & 13,237 & 12,332 & 7,34 \\
\hline
\end{tabular}

Hf t-t - Método Trecho-a-Trecho; Hf ms - Método de Múltiplas Saídas; $\Delta$ Hf - variação (\%).

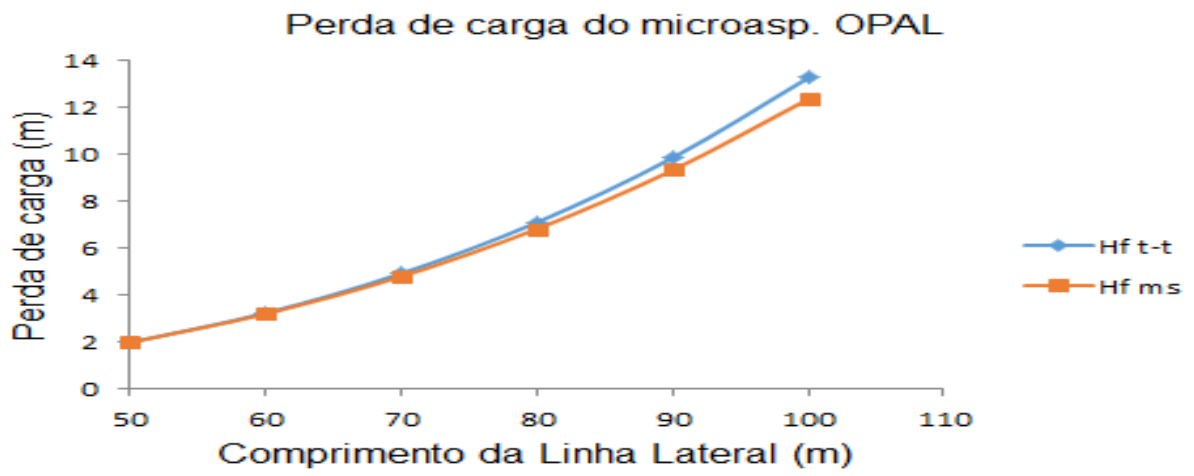

Figura 3. Dinâmica da perda de carga com o aumento do comprimento da linha lateral. 
Tabela 3. Perdas de carga $(\Delta \mathrm{Hf})$ para os dois métodos em função do aumento da pressão de serviço do emissor (PS).

\begin{tabular}{cccc}
\hline PS (mca) & HF t-t (m) & Hf $\mathbf{~ m s ~ ( m ) ~}$ & $\Delta$ Hf (\%) \\
\hline 10 & 8,082 & 7,428 & 8,80 \\
12 & 9,200 & 8,488 & 8,39 \\
14 & 10,226 & 9,500 & 7,64 \\
16 & 11,290 & 10,475 & 7,78 \\
18 & 12,279 & 11,419 & 7,53 \\
20 & 13,237 & 12,332 & 7,34 \\
\hline
\end{tabular}

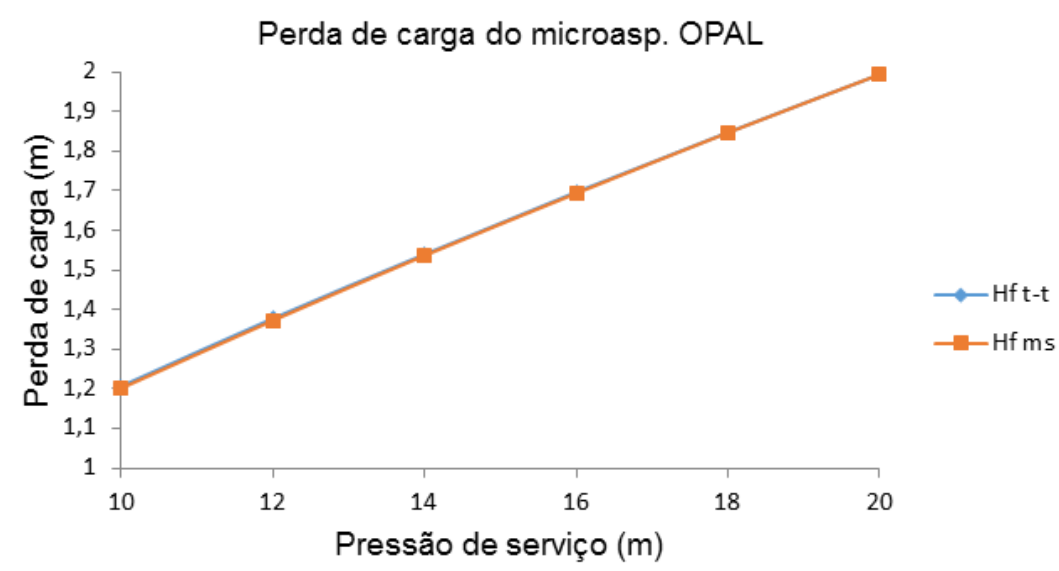

Figura 4. Perda de carga em função do aumento da pressão de serviço do emissor(PS).

A perda de carga determinada pelo método trecho-a-trecho, quando a pressão de serviço (PS) foi aumentada, foi maior que o método de múltiplas saída, entretanto houve um decréscimo nas diferenças apresentadas entre os dois métodos estudados, a maior diferença entre os dois métodos foi de $8,8 \%$.

\section{Conclusões}

Tanto para o aumento do comprimento da linha lateral como para o aumento da pressão de serviço, a perda de carga determinada pelo método trecho-a-trecho foi maior que o método de múltiplas saídas.

\section{Conflito de interesses}

Os autores declaram não haver conflito de interesses.

\section{Referências}

Alazba, A. A.; Mattar, M. A.; Elnesr, M. N.; Amin, M. T. Field assessment of friction head loss and friction correction factor equations. Journal of Irrigation and Drainage Engineering, v. 138, n. 2, p. 166-176, 2012. https://doi.org/10.1061/(ASCE)IR.19434774.0000387

Alves, D. G.; Pinto, M. F.; Damasceno, A. P. A. B.; Salvador, C. A.; Botrel, T. A.; Silva, L. D. B. Análise dos modelos matemáticos utilizados no dimensionamento de emissores do tipo 
microtubos na microirrigação. Irriga, v. 1, n. 2, p. 21-29, 2015. https://doi.org/ 10.15809/irriga.2015v1n2p21

Bombardelli, W. W. A. Perda localizada de carga em conexões utilizadas em sistemas de microirrigação. Piracicaba: Universidade de São Paulo, Escola Superior de Agricultura "Luiz de Queiroz", 2018. (Dissertação de mestrado em Ciências).

Coelho, A. P; Zanini, J. R; Faria, R. T. De; Dalri, A. B; Palaretti, L. F. Comparação de equações para estimativa da perda de carga em tubulação de polietileno. Pesquisa Aplicada \& Agrotecnologia, v. 11, n. 1, p. 25-31, 2018. https://doi.org/10.5935/PAeT.V11.N1.03

Melo, M. C. Irrigação em pastagens por gotejamento subsuperficial e modelagem do movimento de água no solo. Viçosa: Universidade Federal de Viçosa, 2018. (Dissertação de mestrado).
Rettore Neto, O.; Botrel, T. A.; Frizzone, J. A.; Pinto, M. F.; Camargo, A. P. Quantificação do erro na determinação da perda contínua de carga em tubos elásticos. Engenharia Agrícola, v. 33, n. 6, p. 1312-1321, 2013. https://doi.org/10.1590/S0100-691620130 00600023

Rettore Neto, O.; Botrel, T. A.; Frizzone, J. A.; Camargo, A.P. Method for determining friction head loss along elastic pipes. Irrigation Science, v. 32, p. 329-339, 2014. https://doi.org/10.1007/s00271-014-04317

Sampaio, S. C.; Frigo, E. P.; Boas, M. A. V.; Queiroz, M. M. F.; Gomes, B. M.; Mallmann, L. S. Perda de carga em tubulações e conexões conduzindo água residuária da avicultura. Irriga, v. 12, n. 2, p. 225-234, 2007. https://doi.org/10.15809/irriga.2007v12n2 p225-234

Informação da Licença: Este é um artigo Open Access distribuído sob os termos da Licença Creative Commons Attribution, que permite uso irrestrito, distribuição e reprodução em qualquer meio, desde que a obra original seja devidamente citada. 